\title{
SRLG inference in OSPF for improved reconvergence after failures
}

\author{
Bart Puype ${ }^{1}$, Dimitri Papadimitriou ${ }^{2}$, Goutam Das ${ }^{1}$, \\ Didier Colle ${ }^{1}$, Mario Pickavet ${ }^{1}$ and Piet Demeester ${ }^{1}$, \\ ${ }^{1}$ University of Ghent, Department of Information Technology (INTEC), \\ Gaston Crommenlaan 8 bus 201, B-9050 Gent, Belgium \\ \{bart.puype, gdas, dcolle, mpick, demeester\}@intec.ugent.be \\ ${ }^{2}$ Alcatel-Lucent Bell, Copernicuslaan 50, B-2018 Antwerpen, Belgium \\ dimitri.papadimitriou@alcatel-lucent.com
}

\begin{abstract}
The ECODE FP7 project researches cognitive routing functions in future networks. We demonstrate machine learning augmented OSPF routing which infers SRLGs from network failure history. Inferred SRLGs are used to improve OSPF convergence and recovery times during subsequent (multiple) network failures.
\end{abstract}

Keywords: OSPF, machine learning, cognitive routing, network recovery

\section{Overview}

As part of the Future Internet Research and Experimentation (FIRE) initiative, the ECODE FP7 (Experimental COgnitive Distributed Engine) project [1] designs and experiments cognitive routing system functionality. For this purpose, online distributed machine learning techniques are developed to improve network performance. Specifically for this demonstration, the Open Shortest Path First (OSPF) link state routing protocol [2] and its functionality to recover from network failures using IP re-routing are considered. A machine learning component was developed and implemented, which allows the OSPF to anticipate multiple network failures, and speed-up routing convergence and recovery of connectivity.
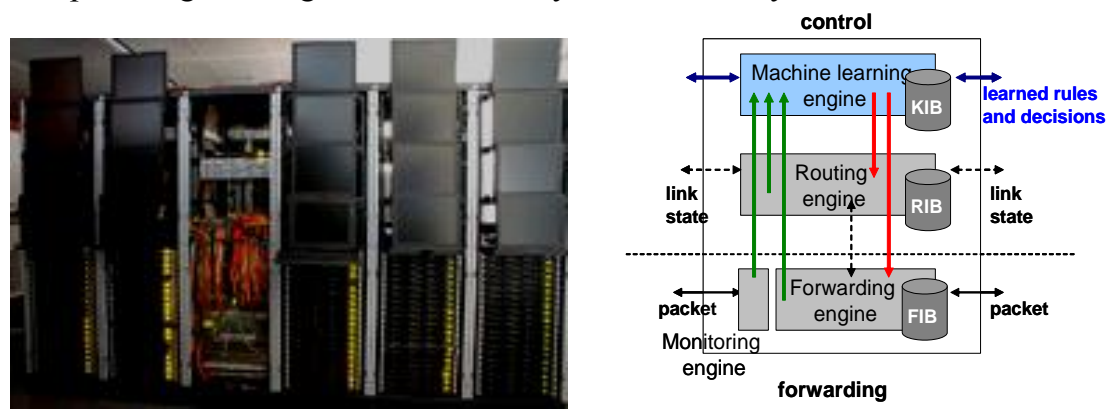

Fig. 1. IBBT ilab.t facility (left); routing system functional overview (right) 
This functionality was developed and tested on the IBBT ilab.t facility (Fig. 1, left), a network emulation platform allowing for easy setup and execution of networking experiments. The ECODE cognitive routing platform uses the eXtensible Open Routing Platform [3], with the machine learning component implemented as a XORP process (Fig. 1, right). The machine learning engine augments control plane functionality, and interacts with the routing and forwarding engine.

\section{SRLG inference}

Layered networks suffer from multiple concurrent upper layer failures caused by failure of a lower layer resource. A set of network links relying on a lower layer resource is called a shared risk link group (SRLG). The SRLG inference algorithm running in the machine learning engine receives link failure event information from the OSPF protocol running in the local routing engine. From the failure history, the algorithm can learn and identify SRLGs [4]. These are then passed to the OSPF instance. When the OSPF protocol receive a link state update indicating a link failure, it can anticipate further link failures from SRLG information, leading to faster network recovery.

\section{Demonstrated functions}

We demonstrate SRLG inference by running the machine learning augmented OSPF on an emulated network. Each node runs a separate augmented OSPF instance, demonstrating distributed machine learning. The setup visualizes network connectivity by displaying a graph model of the network, as well as showing several video streams transported over the network, allowing spectators to verify the impact on network connectivity of multiple link failures. We compare standard OSPF which offers slow, piece-wise recovery of connectivity, versus SRLG inference augmented OSPF operation which reroutes and recovers for all links in an entire SRLG at once. A short video overview of the demonstration setup is available at [5].

Acknowledgments. This research work is (partially) funded by the European Commission (EC) through the ECODE project (INFSO-ICT-223936) part of the European Seventh Framework Programme (FP7).

\section{References}

1. ECODE FP7 project, http://www.ecode-project.eu/

2. J. Moy, OSPF Version 2, RFC 2328, Internet Engineering Task Force (IETF), April 1998.

3. XORP, eXtensible Open Router Platform, http://www.xorp.org/

4. G. Das, D. Papadimitriou, W. Tavernier, D. Colle, T. Dhaene, M. Pickavet, and P. Demeester, Link State Protocol data mining for shared risk link group detection, Proc. of 19th International Conference on Computer Communications and Networks (ICCCN 2010), Zurich, Switzerland, 2-5 August 2010.

5. Demonstration video, http://users.ugent.be/ bpuype/pub/fia-ghent/ 